\title{
Aspirin upregulates the expression of neuregulin 1 and survivin after focal cerebral ischemia/reperfusion in rats
}

\author{
FAN HE ${ }^{1}$, FANG $\mathrm{QU}^{1}$ and FULIN SONG ${ }^{2}$ \\ Departments of ${ }^{1}$ Neurology, and ${ }^{2}$ Pathology, Shenyang Northern Hospital, Shenyang 110016, P.R. China
}

Received October 21, 2011; Accepted December 27, 2011

DOI: $10.3892 /$ etm.2012.450

\begin{abstract}
Neuregulin 1 (NGR1) and survivin have been shown to be neuroprotective. However, the link between their expression and aspirin in the treatment of cerebral ischemia remains unclear. Here, we investigated the effect of aspirin on NGR1 and survivin expression after focal cerebral ischemia/reperfusion in rats. Sprague Dawley rats were randomly divided into an aspirin treatment group $(n=40)$ and a control group $(n=40)$. Each group was further divided into five subgroups according to the time after reperfusion. A middle cerebral artery model was established by an occlusion suture. At 24 h, 3, 5 and 7 days after reperfusion, the Bederson neurological deficit scores were $1.47 \pm 0.11,1.22 \pm 0.08,0.85 \pm 0.15$ and $0.59 \pm 0.12$ in the treatment group, and $1.87 \pm 0.18,1.45 \pm 0.14,1.05 \pm 0.08$ and $0.75 \pm 0.15$ in the control group, respectively, indicating a significant difference at each time point $(\mathrm{P}<0.05)$. In the infarct center, the number of NGR1- and survivin-positive cells reached the maximum at $6 \mathrm{~h}$ and decreased gradually to a minimum at 7 days, while in the peri-infarct area, the number was few at $6 \mathrm{~h}$, peaked at 3 days and then was reduced gradually with significant differences between the two time points $(\mathrm{P}<0.05)$. There were more NGR1and survivin-positive cells in the treatment group compared to the control group $(\mathrm{P}<0.05)$. In conclusion, the neuroprotective effect of aspirin is at least partly mediated by the upregulation of NGR1 and survivin expression after ischemia.
\end{abstract}

\section{Introduction}

Neuregulin 1 (NGR1) is a signaling protein which plays an important role in the nervous system development. It is mainly expressed in neurons, glial cells and organs, such as the heart, liver, stomach, lung, kidney and spleen. Moreover, NGR1 is implicated in numerous nervous system diseases. In models of cerebral ischemia, exogenous NGR1 inhibits neuronal apoptosis and reduces the volume of cerebral infarction (1-6). Survivin is a well-known anti-apoptotic protein that inhibits

Correspondence to: Dr Fang Qu, Department of Neurology, Shenyang Northern Hospital, Shenyang 110016, P.R. China E-mail: fangqu168@hotmail.com

Key words: cerebral ischemia/reperfusion, aspirin, neuregulin 1, survivin the activities of caspase- 3 and caspase-7. Only recently has the role of survivin in cerebral ischemia become appreciated (7). Aspirin has neuroprotective effects, including the inhibition of apoptosis of ischemic brain cells $(8,9)$. However, the link between NGR1 and survivin expression and aspirin in the treatment of cerebral ischemia remains unclear. Here, we investigated the effect of aspirin on the expression patterns of NGR1 and survivin after focal cerebral ischemia/reperfusion in rats to further explore the neuroprotective mechanism of aspirin.

\section{Materials and methods}

Animals and groups. A total of 80 healthy male Sprague Dawley rats (weight 240-280 g) were provided by the Medical Animal Laboratory, Shenyang Military General Hospital. Animals were fasted for $12 \mathrm{~h}$ prior to surgery with water offered ad libitum. The rats were randomly divided into a treatment group (aspirin, $\mathrm{n}=40$ ) and a control group (L-lysine, $\mathrm{n}=40$ ). Each group was further divided into five equal subgroups according to the time since reperfusion $(6 \mathrm{~h}, 24 \mathrm{~h}, 3$, 5 and 7 days, $n=8$ in each group). Aspirin powder was provided by the Pharmaceutical Factory of Shenyang Pharmaceutical University, and diluted with L-lysine before use.

Establishing the cerebral ischemia/reperfusion (CI/RP) model. Cerebral ischemia was established with an occlusion suture as previously described $(10,11)$. Two hours after the induction of cerebral ischemia, the suture was pulled back slowly $\sim 2 \mathrm{~mm}$ for reperfusion. Aspirin powder ( $80 \mathrm{mg} / \mathrm{kg}$ body weight) dissolved in $1.5 \mathrm{ml}$ of $10 \%$ L-lysine saline solution was injected intraperitoneally at the time of reperfusion and once each morning thereafter. Brain samples were obtained at 6 h, 24 h, 3, 5 and 7 days after reperfusion. In the control group, intraperitoneal infusion of $1.5 \mathrm{ml}$ of $10 \%$ L-lysine solution was administered at the same time points, and brain samples were obtained as in the treatment group. No sham-operated group was set since sham operation on cervical vessels does not cause cerebral infarction in the supplied area. Supplementary rats were available according to a protocol of randomization and grouping when rats were excluded or died during the experiment. The evaluation of limb function was carried out according to the Bederson scoring system (12) every day after the CI/RP surgery to assess the recovery of neurological function. The scoring formula was as follows: 0, without any neurologic deficit; 1, dysfunction of left forelimb extension; 2, walking towards the left; 3 , circling 
Table I. Bederson scores for the rats in the two groups at different time points after CI/RP (mean \pm SD).

\begin{tabular}{lccccc}
\hline Group & $\mathrm{n}$ & $6 \mathrm{~h}$ & $24 \mathrm{~h}$ & 3 days & 5 days \\
\hline Treatment & 40 & $1.97 \pm 0.09^{\mathrm{a}}$ & $1.47 \pm 0.11^{\mathrm{c}}$ & $1.22 \pm 0.08^{\mathrm{c}}$ & $0.85 \pm 0.15^{\mathrm{c}}$ \\
Control & 40 & $1.94 \pm 0.13$ & $1.87 \pm 0.18$ & $1.45 \pm 0.14$ & $1.05 \pm 0.08$ \\
$\mathrm{t}$ & & 0.89 & 5.36 & 4.03 & $3.33 \pm 0.12^{\mathrm{b}}$ \\
\hline
\end{tabular}

Compared to the control group: ${ }^{\mathrm{a}} \mathrm{P}>0.05 ;{ }^{\mathrm{b}} \mathrm{P}>0.05 ;{ }^{\mathrm{c}} \mathrm{P}>0.01$.

Table II. NGR1 expression in different infarct areas at different time points after CI/RP in the two groups (n=40; cells/field).

\begin{tabular}{|c|c|c|c|c|c|c|}
\hline \multirow[t]{2}{*}{ Time after CI/RP } & \multicolumn{3}{|c|}{ Infarct center } & \multicolumn{3}{|c|}{ Peri-infarct area } \\
\hline & Treatment group & Control group & $\mathrm{t}$ & Treatment group & Control group & $\mathrm{t}$ \\
\hline $6 \mathrm{~h}$ & $43.4 \pm 4.7$ & $40.30 \pm 5.6$ & $2.68^{\mathrm{a}}$ & $16.7 \pm 2.6$ & $15.3 \pm 2.2$ & $2.59^{\mathrm{a}}$ \\
\hline $24 \mathrm{~h}$ & $33.1 \pm 3.4$ & $31.30 \pm 3.2$ & $2.44^{\mathrm{a}}$ & $25.8 \pm 3.5$ & $23.9 \pm 2.7$ & $2.72^{\mathrm{a}}$ \\
\hline 3 days & $25.4 \pm 2.2$ & $23.11 \pm 1.9$ & $4.57^{b}$ & $31.4 \pm 4.5$ & $27.6 \pm 4.1$ & $3.95^{\mathrm{b}}$ \\
\hline 5 days & $16.4 \pm 2.7$ & $14.90 \pm 2.2$ & $2.72^{\mathrm{a}}$ & $26.3 \pm 3.4$ & $24.7 \pm 2.1$ & $2.53^{\mathrm{a}}$ \\
\hline 7 days & $6.9 \pm 2.1$ & $5.80 \pm 1.7$ & $2.57^{\mathrm{a}}$ & $19.3 \pm 2.6$ & $17.8 \pm 2.4$ & $2.68^{\mathrm{a}}$ \\
\hline
\end{tabular}

${ }^{\mathrm{a}} \mathrm{P}<0.05 ;{ }^{\mathrm{b}} \mathrm{P}<0.01$.

Table III. Survivin expression in different infarct areas at different time points after CI/RP in the two groups ( $\mathrm{n}=40$; cells/field).

\begin{tabular}{|c|c|c|c|c|c|c|}
\hline \multirow[t]{2}{*}{ After CI/RP } & \multicolumn{3}{|c|}{ Infarct center } & \multicolumn{3}{|c|}{ Peri-infarct area } \\
\hline & Treatment group & Control group & $\mathrm{t}$ & Treatment group & Control group & $\mathrm{t}$ \\
\hline $6 \mathrm{~h}$ & $41.2 \pm 3.9$ & $38.8 \pm 4.7$ & $2.49^{\mathrm{a}}$ & $17.4 \pm 2.8$ & $15.8 \pm 3.7$ & $2.18^{\mathrm{a}}$ \\
\hline $24 \mathrm{~h}$ & $31.1 \pm 4.4$ & $28.8 \pm 3.6$ & $2.56^{\mathrm{a}}$ & $26.7 \pm 2.6$ & $25.2 \pm 3.4$ & $2.21^{\mathrm{a}}$ \\
\hline 3 days & $21.8 \pm 3.7$ & $18.6 \pm 4.5$ & $3.47^{\mathrm{b}}$ & $33.4 \pm 3.7$ & $30.7 \pm 2.5$ & $3.82^{\mathrm{b}}$ \\
\hline 5 days & $15.3 \pm 1.7$ & $13.7 \pm 3.5$ & $2.60^{\mathrm{a}}$ & $27.3 \pm 3.6$ & $25.1 \pm 4.6$ & $2.38^{\mathrm{a}}$ \\
\hline 7 days & $8.9 \pm 3.4$ & $7.3 \pm 2.1$ & $2.53^{\mathrm{a}}$ & $20.3 \pm 1.6$ & $19.2 \pm 2.6$ & $2.28^{\mathrm{a}}$ \\
\hline
\end{tabular}

${ }^{\mathrm{a}} \mathrm{P}<0.05 ;{ }^{\text {b }} \mathrm{P}<0.01$.

towards the left; 4 , were unable to walk or in a coma. Rats with scores of 1-3 were assumed to be successful CI/RP models and others were excluded from the study.

Immunohistochemical analysis. Immunohistochemical analysis was performed as described previously (13). At each experimental time point, after intraperitoneal anesthesia with an overdose of chloral hydrate $(10 \%)$, the thoracic cavity was immediately opened to expose the heart. Perfusion then proceeded rapidly with $200 \mathrm{ml}$ isotonic saline and $200 \mathrm{ml} 4 \%$ paraformaldehyde in PBS injected into the left ventricle. The brain was extracted after decapitation and fixed in $4 \%$ paraformaldehyde for $24 \mathrm{~h}$. Brain tissues were incised along the coronal plane, and then conventionally dehydrated and embedded in paraffin. Serial sections were cut at a thickness of $4 \mathrm{~mm}$, and dried at $37^{\circ} \mathrm{C}$ in an incubator overnight. Immunohistochemical staining was performed using S-P immunohistochemical and diaminobenzidene (DAB) kits (Fujian Maixin Biological, Fujian, China). NGR1 polyclonal antibody (sc-348) was from
Santa Cruz Company, USA. Positive cells showed as brown particles in the cytoplasm or nucleus under a light microscope. Five different high power fields $(10 \times 40)$ were randomly selected blindly in positive cell areas (the temporal parietal cortex was selected in the penumbra area and the temporal parietal subcortex was selected in the infarct center), and the number of positive cells in each visual field was counted (14).

Statistical analyses. Statistical Package for the Social Sciences (SPSS) 12 software was used for statistical analyses. Data are represented as the means \pm standard deviation (SD). Variance analysis and the t-test were used for tests of significance. A $\mathrm{P}$-value $<0.05$ was considered to denote statistical significance.

\section{Results}

After induction of CI/RP, neurological deficit was observed in both the treatment and control groups, and the main manifestations were as follows: adduction of the contralateral forelimb; 

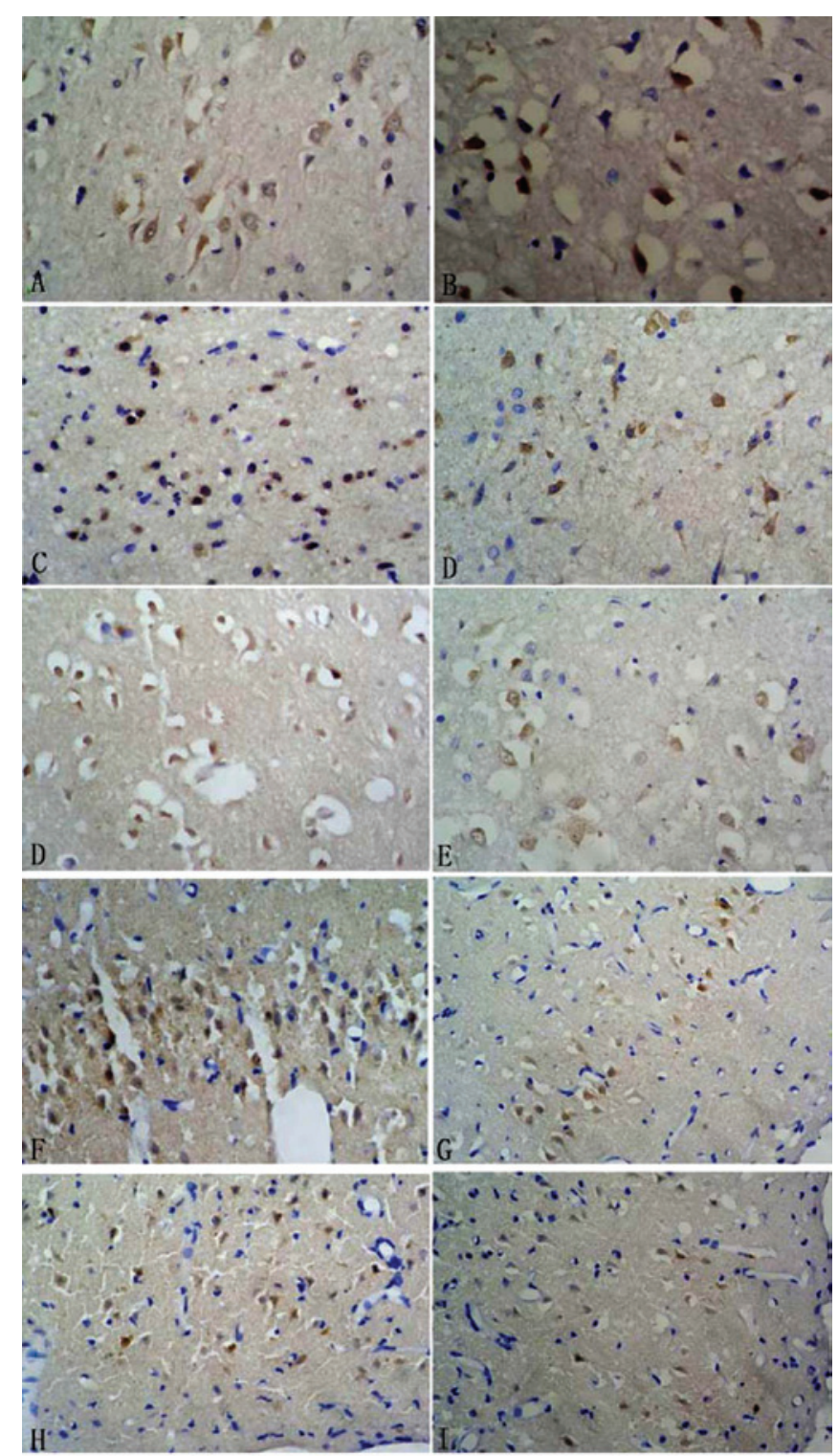

Figure 1. Intracerebral NGR1 expression at different sites after CI/RP in rats. Brown staining represents positive NGR1 expression. (A) Infarct center in the treatment group (6 h). (B) Infarct center in the control group (6 h). (C) Infarct center in the treatment group (24 h). (D) Infarct center in the control group (24 h). (E) Infarct center in the treatment group (3 days). (F) Infarct center in the control group (3 days). (G) Peri-infarct area (cortical pyramidal layer) in the treatment group (3 days). (H) Peri-infarct area (cortical pyramidal layer) in the control group (3 days). (I) Peri-infarct area (cortical pyramidal layer) in the treatment group (5 days). (J) Peri-infarct area (cortical pyramidal layer) in the control group (5 days). Magnification, $\mathrm{x} 400$.

internal rotation of the shoulder; the left forelimb could not straighten, or it was held close to the chest while lifting the tail; sinistral circular motion; dumping; and decreased muscle tension while walking. At $6 \mathrm{~h}$ after reperfusion, neurological deficit scores were assessed again in the two groups, compared and examined by t-test. No significant difference was found between the two groups $(\mathrm{P}>0.05)$. Twenty-four hours after $\mathrm{CI} / \mathrm{RP}$ surgery, neurological function was restored gradually in both the treatment and control groups, although a better recovery was found in the treatment group. There were statistically significant differences in Bederson scores between the two groups at each time point after $\mathrm{CI} / \mathrm{RP}(\mathrm{P}<0.05$; Table I).
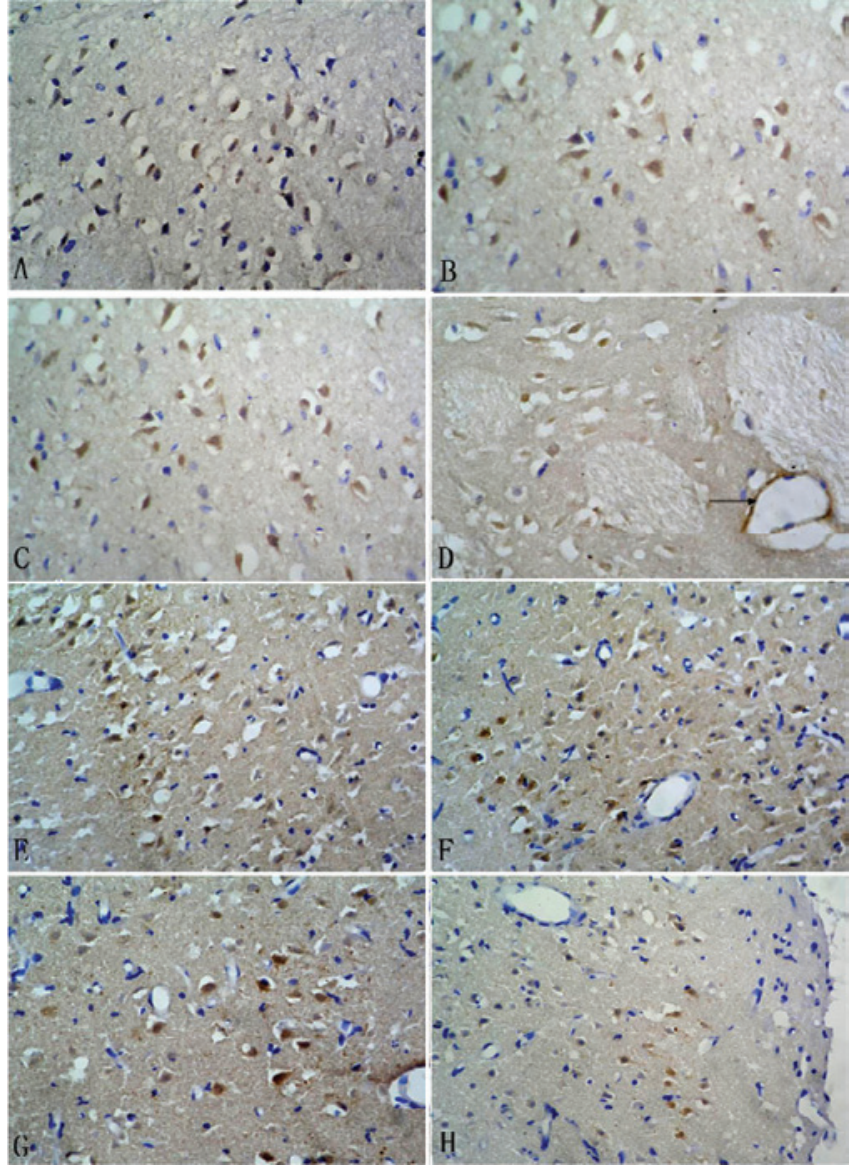

Figure 2. Survivin expression in different infarct areas in CI/RP rats. Brown staining represents positive survivin expression. (A) Infarct center in the treatment group (6 h). (B) Infarct center in the control group (6 h). (C) Infarct center in the treatment group ( 3 days). (D) Infarct center in the control group (3 days). Survivin expression was also detected in small vascular endothelial cells (indicated by arrow). (E) Peri-infarct area (cortical pyramidal layer) in the treatment group (3 days). (F) Peri-infarct area (cortical pyramidal layer) in the control group (3 days). (G) Peri-infarct area (cortical pyramidal layer) in the treatment group (5 days). (H) Peri-infarct area (cortical pyramidal layer) in the control group (5 days). Magnification, $\mathrm{x} 400$.

After CI/RP, NGR1 expression was detected by immunohistochemistry in rat brain tissues in both the treatment and control groups. NGR1 was mainly expressed in the nucleus and cytoplasm of neurons in the infarct side. In the infarct center, NGR1 was expressed strongly $6 \mathrm{~h}$ after CI/RP, and the number of NGR1-positive cells was at a maximum at $6 \mathrm{~h}$ and decreased gradually. In the peri-infarct area, the number of NGR1-positive cells was small at $6 \mathrm{~h}$, peaked at 3 days and then decreased gradually. Compared to the control group, more NGR1-positive cells were detected in the treatment group at each time point, with significant differences between both time points $(\mathrm{P}<0.05$; Table II and Fig. 1).

The expression pattern of survivin was similar to that of NGR1 after CI/RP and, also, it was mainly expressed in the nucleus and cytoplasm of neurons in the infarct side. In addition, survivin expression was found in small vascular endothelial cells 3 days after CI/RP. In the infarct center, survivin was expressed strongly $6 \mathrm{~h}$ after CI/RP, and the number of survivin-positive cells was maximal at $6 \mathrm{~h}$ and decreased gradually. In the peri-infarct area, the number of survivin-positive cells was small at $6 \mathrm{~h}$, peaked at 3 days and 
decreased gradually. Compared to the control group, more survivin-positive cells were detected in the treatment group at each time point, with significant differences between the two time points $(\mathrm{P}<0.05$; Table III and Fig. 2).

\section{Discussion}

Emerging evidence suggests the potential neuroprotective role of NGR1 in cerebral ischemia. Increased NGR1 expression has been found in neurons of ischemic penumbra, although it is not expressed in astrocytes or microglia cells (15). In addition, injection of exogenous NGR1 into the brain or carotid artery reduces significantly the volume of the infarction and the number of TUNEL-positive and caspase-3-positive cells (1-6). Survivin is the strongest apoptosis inhibitory protein known, and it directly or indirectly inhibits the caspase-dependent or -independent apoptosis pathways (16).

Few reports have addressed the expression patterns of NGR1 and survivin after cerebral ischemia with exogenous stimulation (including administration of drugs). In the present study, the expression patterns of NGR1 and survivin in brain tissues after CI/RP alone, or in combination with aspirin administration, were examined by immunohistochemistry. The results demonstrated that NGR1 and survivin were mainly expressed in the nucleus and cytoplasm of neurons in the infarct hemisphere after $\mathrm{CI} / \mathrm{RP}$, transiting from the infarct center to the peri-infarct area. In the infarct center, NGR1 and survivin were expressed most significantly $6 \mathrm{~h}$ after CI/RP, and reached a peak after 3 days in the peri-infarct area. With aspirin treatment, the number of survivin- and NGR1-positive cells increased, but the time of peak expression did not change. In the early stage of CI/RP (within $6 \mathrm{~h}$ post-CI/RP), the infarct center was severely ischemic without complete necrosis in most of the neurons, therefore NGR1 and survivin expression remained high. However, the peri-infarct area was only mildly ischemic, and so there were only a few NGR1- and survivinpositive cells. As time passed (after 3 days), massive necrosis of neurons was found in the infarct center, and the expression of NGR1 and survivin subsided gradually. However, edema and ischemia in the peri-infarct area became aggravated, and the number of NGR1- and survivin-positive cells increased. At 7 days, NGR1- and survivin-positive cells were fewer, which may be related to the increased necrosis in the peri-infarct area and the restoration of cerebral blood supply. Taken together, our data suggest that ischemia is a strong stimulus to induce the expression of endogenous NGR1 and survivin. NGR1 and survivin expression may be further upregulated by exogenous aspirin. NGR1 and survivin may play neuroprotective roles through the inhibition of apoptosis, providing a mechanism by which aspirin exhibits its neuroprotective effects.

Conway et al (7) reported that survivin was also expressed in cerebral pia mater and small blood vessels. Our experiment also confirmed that survivin is expressed in a portion of the vascular endothelial cell infract center and peri-infarct area, but NGR1 was not expressed in these areas. These data indicate that survivin promotes angiogenesis (7). Indeed, survivin is upregulated by VEGF and plays an anti-apoptosis role in the process of angiogenesis (17). Plate et al (18) reported that after middle cerebral artery occlusion, the mRNA expression of vascular endothelial growth factor (VEGF) was detected in gliocytes and macrophages in the perivascular ischemic penumbra and reached a peak after 2 days (18), which appropriately coincides with post-ischemic angiogenesis in the infarct center (19). Although VEGF may facilitate the leakage of capillary blood and destroy the blood-brain barrier to aggravate brain edema, its effect on angiogenesis may be beneficial to long-term recuperation (20). Therefore, we propose that the concomitant upregulation of VEGF and survivin may help maintain a balance and promote maximal neuroprotective effects against cerebral ischemia/reperfusion.

\section{References}

1. Guo WP, Wang J, Li RX and Peng YW: Neuroprotective effects of neuregulin-1 in rat models of focal cerebral ischemia. Brain Res 1087: 180-185, 2006.

2. Li Q, Li Z, Mei Y and Guo Y: Neuregulin attenuated cerebral ischemia-reperfusion injury via inhibiting apoptosis and upregulating aquaporin-4. Neurosci Lett 443: 155-159, 2008.

3. Li Q, Zhang R, Guo YL and Mei YW: Effect of neuregulin on apoptosis and expressions of STAT3 and GFAP in rats following cerebral ischemic reperfusion. J Mol Neurosci 37: 67-73, 2009.

4. Shyu WC, Lin SZ, Chiang MF, Yang HI, Thajeb P and Li H: Neuregulin-1 reduces ischemia-induced brain damage in rats. Neurobiol Aging 25: 935-944, 2004.

5. Xu Z, Croslan DR, Harris AE, Ford GD and Ford BD: Extended therapeutic window and functional recovery after intraarterial administration of neuregulin-1 after focal ischemic stroke. J Cereb Blood Flow Metab 26: 527-535, 2006.

6. Xu Z, Jiang J, Ford G and Ford BD: Neuregulin-1 is neuroprotective and attenuates inflammatory responses induced by ischemic stroke. Biochem Biophys Res Commun 322: 440-446, 2004.

7. Conway EM, Zwerts F, Van Eygen V, et al: Survivin-dependent angiogenesis in ischemic brain: molecular mechanisms of hypoxia-induced up-regulation. Am J Pathol 163: 935-946, 2003.

8. Legos JJ, Mangoni AA, Read SJ, et al: Programmable microchip monitoring of post-stroke pyrexia: effects of aspirin and paracetamol on temperature and infarct size in the rat. J Neurosci Methods 113: 159-166, 2002.

9. Zheng Z, Schwab S, Grau A and Berger C: Neuroprotection by early and delayed treatment of acute stroke with high dose aspirin. Brain Res 1186: 275-280, 2007.

10. Arumugam TV, Phillips TM, Cheng A, Morrell CH, Mattson MP and Wan R: Age and energy intake interact to modify cell stress pathways and stroke outcome. Ann Neurol 67: 41-52, 2010.

11. Fan Y, Shen F, Frenzel T, et al: Endothelial progenitor cell transplantation improves long-term stroke outcome in mice. Ann Neurol 67: 488-497, 2010.

12. Bederson JB, Pitts LH, Tsuji M, Nishimura MC, Davis RL and Bartkowski H: Rat middle cerebral artery occlusion: evaluation of the model and development of a neurologic examination. Stroke 17: 472-476, 1986.

13. Leinonen V, Koivisto AM, Savolainen S, et al: Amyloid and tau proteins in cortical brain biopsy and Alzheimer's disease. Ann Neurol 68: 446-453, 2010.

14. Perier C, Bove J, Dehay B, et al: Apoptosis-inducing factor deficiency sensitizes dopaminergic neurons to parkinsonian neurotoxins. Ann Neurol 68: 184-192, 2010.

15. Parker MW, Chen Y, Hallenbeck JM and Ford BD: Neuregulin expression after focal stroke in the rat. Neurosci Lett 334: 169-172, 2002.

16. Shin S, Sung BJ, Cho YS, et al: An anti-apoptotic protein human survivin is a direct inhibitor of caspase-3 and -7. Biochemistry 40: 1117-1123, 2001.

17. O'Connor DS, Schechner JS, Adida C, et al: Control of apoptosis during angiogenesis by survivin expression in endothelial cells. Am J Pathol 156: 393-398, 2000.

18. Plate KH, Beck H, Danner S, Allegrini PR and Wiessner C: Cell type specific upregulation of vascular endothelial growth factor in an MCA-occlusion model of cerebral infarct. J Neuropathol Exp Neurol 58: 654-666, 1999.

19. Marti HJ,Bernaudin M,Bellail A, et al: Hypoxia-induced vascular endothelial growth factor expression precedes neovascularization after cerebral ischemia. Am J Pathol 156: 965-976, 2000.

20. Zhang ZG, Zhang L, Jiang Q, et al: VEGF enhances angiogenesis and promotes blood-brain barrier leakage in the ischemic brain. J Clin Invest 106: 829-838, 2000. 